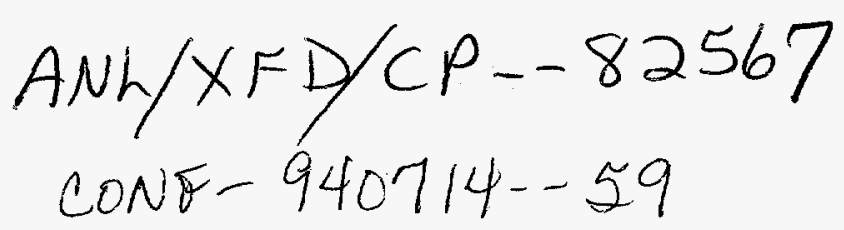

\title{
Anticlastic Curvature Measurements on Unribbed Crystal Optics for Synchrotron Radiation*
}

\author{
J. P. Quintana ${ }^{a}$, Yu Dolin ${ }^{a}$, P. Georgopoulos ${ }^{a}$, and V. I. Kushnir ${ }^{b}$ \\ aDND-CAT Synchrotron Research Center, \\ APS/ANL Sector 5, 9700 S. Cass Avenue, Argonne, IL 60439 \\ ${ }^{b}$ Experimental Facilities Division, Advanced Photon Source, \\ Argonne National Laboratory, Argonne, IL 60439 \\ The Goverment reserves for itself and \\ others acting on its behalf a royalty free, \\ nonexclusive, irrevocable, world-wide \\ license for govermental purposes to publish, \\ distribute, translate, duplicate, exhibit, \\ and perform any such data copyrighted by \\ the contractor.
}

\section{January 1996}

The submitted manuscript has been authored
by a contractor of the U.S. Government
under contract No. W-31-t09-ENG-38.
Accordingly, the U.S. Government retains a
nonexclusive, royalty-free license to publish
or reproduce the published form of this
contribution, or allow others to do so, for
U.S. Government purposes.

\section{DISCLAIMER}

\begin{abstract}
This report was prepared as an account of work sponsored by an agency of the United States Government. Neither the United States Government nor any agency thereof, nor any of their employees, makes any warranty, express or implied, or assumes any legal liability or responsibility for the accuracy, completeness, or usefulness of any information, apparatus, product, or process disclosed, or represents that its use would not infringe privately owned rights. Reference herein to any specific commercial product, process, or service by trade name, trademark, manufacturer, or otherwise does not necessarily constitute or imply its endorsement, recommendation, or favoring by the United States Government or any agency thereof. The views and opinions of authors expressed herein do not necessarily state or reflect those of the United States Government or any agency thereof.
\end{abstract}

Presented at the 5th International Conference on Synchrotron Radiation Instrumentation, Stony Brook, NY, July 18-22, 1994, and published in Rev. Sci. Instrum. 66(2), February 1995, 2188-2189.

\footnotetext{
*This work supported by the U.S. Department of Energy, Basic Energy Sciences-Materials Sciences, under contract \#W-31-109-ENG-38.
} 


\title{
Anticlastic curvature measurements on unribbed crystal optics for synchrotron radiation
}

\author{
J. P. Quintana, Yu. Dolin, and P. Georgopoulos \\ DND-CAT Synchrotron Research Center, APS/ANL Sector 5, Building 400, 9700 South Cass Avenue, \\ Argonne, Illinois 60439 \\ V. I. Kushnir \\ APS/XFD, Bldg. 362, 9700 South Cass Ave., Argonne, Illinois 60439
}

(Presented on 21, July 1994)

\begin{abstract}
Various methods have been proposed for measuring the distortion in perfect crystals using double-crystal methods. The majority of these methods rely on making comparisons between double-crystal rocking curve measurements under the spatial extent of an extended $x$-ray beam. Unless the beam is large and parallel (such as at a synchrotron bending magnet), these methods are not easily scalable to large crystals (e.g., crystal focusing elements for synchrotron beamlines) due to the mechanical inaccuracies inherent in moving the various optical components. We present a method based on a scanning source which simplifies the problems in scaling double-crystal methods to large optics. In addition, results using this method are presented on a ribless sagittal focusing Si(111) crystal demonstrating that the anticlastic deviation can be made to be less than $\pm 1 \mathrm{~s}$ of arc over a 1-cm-long section parallel to the sagittal axis. (c) 1995 American Institute of Physics.
\end{abstract}

\section{INTRODUCTION}

Double crystal $x$-ray diffractometry has been shown to be an extremely useful tool in determining the macroscopic strain in semiconductor single crystals (e.g., curvature left over from polishing induced strain. ${ }^{1-4}$ In particular, the ability to accurately map the curvature in large single crystals is particularly important in developing focusing $\mathrm{x}$-ray optics for synchrotron radiation (e.g., crystals for sagittal focusing). While the width of the $x$-ray rocking curve is related to macroscopic strain, the most direct method of measuring the curvature is by correlating the position of the $\mathrm{x}$-ray singlecrystal rocking curve at different points along the crystal. This can be done either by using the fact that two crystals in the $(n,-n)$ geometry are dispersive if one crystal is bent ${ }^{4}$ and the other flat or by using topographic techniques with an extend parallel beam. In the latter method, one-dimensional and two-dimensional detectors are quite useful, and the beam can be made extremely parallel and monochromatic by using appropriate crystal optics. ${ }^{2}$ However, the expense of multidimensional $x$-ray detectors may make these methods impractical especially when the high spatial resolution afforded by these detectors is not required. Also, topographic methods are not easily adaptable to large crystal areas since the distorted crystal must be translated along the beam and the angular relation between the reference and test crystal must be preserved to sub-arcsecond accuracy. This can be particularly problematic if the translated crystal is attached to a heavy mechanical mechanism such as a crystal bender which is being evaluated for performance considerations. The purpose of this work is to present a cost effective, reliable, and stable method for mapping $\mathrm{x}$-ray rocking curves that is useful for large crystal areas.

\section{EXPERIMENTS AND RESULTS}

In order to measure the curvature in bent $\mathrm{Si}(111)$ single crystals for focusing, we use an $(n,-n)$ double-crystal ar- rangement with the detector and $\mathrm{Cu} x$-ray tube mounted to a scanning $x-z$ stage. The experimental arrangement for the scanning source double-crystal goniometer (SSDCG) is shown in Fig. 1. A $0.5 \mathrm{~mm}$ pinhole is used on the tube so that the $\mathrm{Si}(333)$ reflection can discriminate between the $K \alpha$ doublet. We use a 100 -mm-diam, 6-mm-thick float-zone Si(111) wafer as a reference first crystal set to diffract the $\mathrm{Si}(333)$ from $\mathrm{Cu} K \alpha_{1}$. As the $\mathrm{x}$-ray beam is translated, the first crystal will diffract that portion of the beam which fits within the local $x$-ray rocking curve. Consequently, the parallelism of the beam at different locations as it comes off the first crystal is determined by the crystal uniformity and not the parallel-

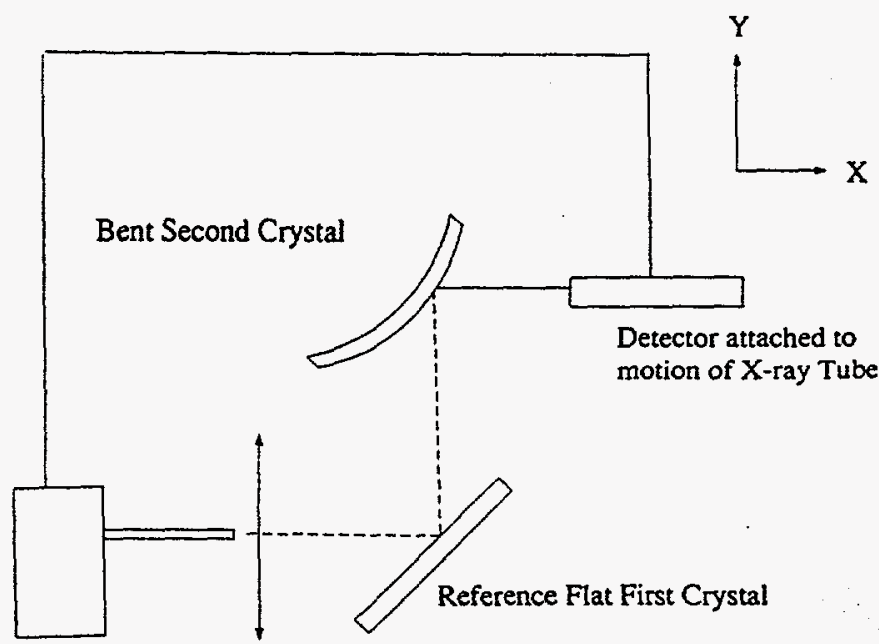

$X$-ray Tube mounted to $x-z$ Translation Stage

FIG. 1. Schematic of a scanning source double-crystal goniometer. The source translates it the $y-z$ plane sweeping over the entire reference crystal. The detector holder is mounted to the same translation stages so the detector constantly tracks the exit beam. 


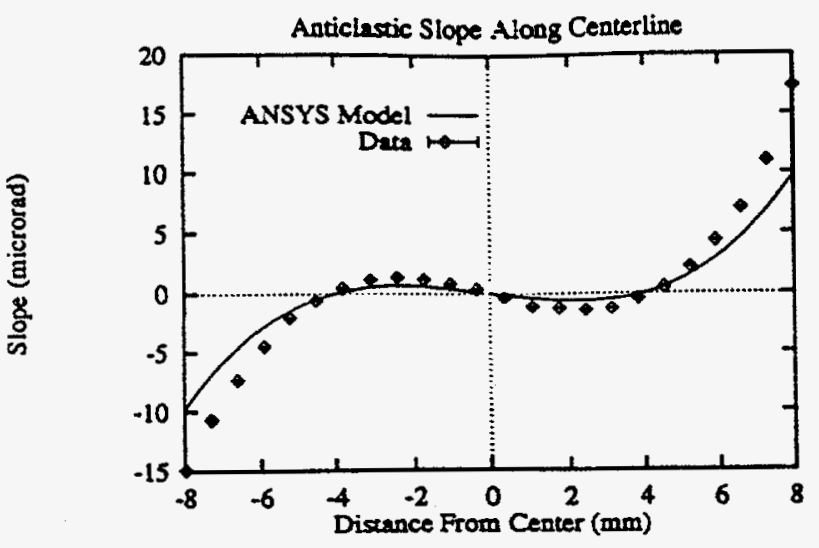

FIG. 2. Slope along the anticlastic direction of an unribbed Si(111) crystal of dimensions $51.18 \times 73.46 \times 0.38 \mathrm{~mm}$ with monolithic thick supports. Crystal was bent to a radius of $2.02 \mathrm{~m}$ (data and model).

ism of the motion of the $\mathrm{x}$-ray tube scanning stages. X-ray rocking curves are taken using a tangent drive goniometer constructed from a commercial mirror mount system driven by linearly encoded inchworms. The goniometer rests on a rotary table for coarse manual adjustments. Using the inchworms in the encoded mode, the system provides a step size of 0.8 arcsec over a $\pm 2^{\circ}$ range. The system stability is verified by plotting the position of the rocking curve along a flat $\mathrm{Si}(111) \mathrm{FZ}$ second crystal and ensuring that there is no change in the position of the Si(333) rocking curve while the beam is scanned. After verification, a crystal bender along with a crystal designed to minimize anticlastic curvature ${ }^{5,6}$ is mounted onto the second crystal drive. The test crystal was machined out of a single monolithic piece of silicon to create a rectangular crystal $51.18 \times 73.46 \times 0.38 \mathrm{~mm}$ with thick supports. Since the amount of anticlastic curvature is strongly dependent on the actual boundary conditions causing the bend, the thick monolithic supports are used to insure that the boundary conditions along the bent portion of the crystal are independent of the actual bending mechanism. Figure 2 shows a plot of the fitted position of the $\mathrm{Si}(333)$ rocking curve along the crystal centerline taken with the SSDCG when the crystal is bent to $2.02 \mathrm{~m}$. The model calculation was performed with ANSYYS using "clamped" boundary conditions. ${ }^{5,6}$ The anticlastic deviation is less than $\pm 1 \mathrm{~s}$ of arc over the central $1-\mathrm{cm}$-long section parallel to the sagittal axis.

We have presented data from a scanning source double crystal goniometer (SSDCG) that has been used to measure the anticlastic curvature on a ribless bent rectangular crystal. The results confirm the "golden ratio method"5,6 of anticlastic curvature minimization and demonstrate that practical optics can be made using this technique. While the crystal tested has a deviation less than $\pm 1 \mathrm{~s}$ of arc over a $1-\mathrm{cm}-$ long section parallel to the sagittal axis, a larger effective length is achievable by increasing the crystal size while preserving the crystal's aspect ratio.

This work was sponsored by the State of Illinois through Technology Challenge Grant No. 92-82139 and the E. I. DuPont de Nemours and Co. through their support of the DUNU Synchrotron Research Center.

' K. Godwod, A. T. Nagy, and Z. Rek, Phys. Status Solidi A 34, 705 (1976).

${ }^{2}$ D. Gao, S. W. Wilkins, and A. W. Stevenson, Rev. Sci. Instrum. 64, 2561 (1993).

${ }^{3}$ D. Gao, T. J. Davis, S. W. Wilkins, and A. Pogany, Rev. Sci. Instrum. 64, 1831 (1993)

${ }^{4}$ B. G. Cohen and M. W. Focht, Solid-State Electron. 13, 105 (1970).

${ }^{5}$ V. I. Kushnir, J. P. Quintana, and P. Georgopoulos, Nucl. Instrum. Methods A 328, 588 (1993).

${ }^{6}$ J. P. Quintana, V. I. Kushnir, and P. Georgopoulos, Mater. Res. Soc. Symp. Proc. 307, 349 (1993). 